\title{
Monopolistic unions, Brainard uncertainty, and optimal monetary policy
}

\author{
By Timo Henckel
}

Centre for Applied Macroeconomic Analysis, Australian National University, Canberra ACT 0200, Australia; e-mail: timo.henckel@anu.edu.au

\begin{abstract}
Some authors have argued that multiplicative uncertainty may benefit society as the cautionary motive reduces the inflation bias. However, when there are nonatomistic wage setters, higher multiplicative uncertainty may raise the wage premium and unemployment and thus reduce welfare. Furthermore, since central bank preferences also affect the wage premium, delegating policy to an independent central banker with an optimal degree of conservatism cannot deliver a second-best outcome.
\end{abstract}

JEL classifications: E52, J51.

\section{Introduction}

Does society benefit from the delegation of monetary policy to a cautious and conservative central banker? The general presumption is that central bankers are indeed cautious and conservative and that this is a good thing. For example, with reference to Brainard's (1967) landmark article, Blinder argues that,

... under certain conditions, uncertainty about policy multipliers should make policymakers conservative [author's emphasis] in the following specific sense: They should compute the direction and magnitude of their optimal policy move in the way prescribed by Tinbergen-Theil and then do less. (Blinder, 1998, p.11) ${ }^{1}$

With respect to conservatism he states:

Rogoff (1985) cleverly suggested that, if there is an inflationary bias in monetary policy, the cure may lie in the appointment of more 'conservative' central bankers. Now that really does have the ring of truth! Indeed, in the real world the noun 'central banker' practically cries out for the adjective 'conservative'. (Blinder, 1998, p.46)

\footnotetext{
${ }^{1}$ Note that Blinder, in this context, uses the term conservative whereas I adopt the more common term cautionary.
} 
The concepts of caution and conservatism have traditionally been analysed separately. Brainard (1967) argued that caution may be optimal because, in the presence of random multipliers, policy itself injects uncertainty into the economy. The benefits of delegating monetary policy to a conservative central banker was highlighted by Rogoff (1985) as a means of reducing the costly inflation bias resulting from a standard time inconsistency problem à la Kydland and Prescott (1977) and Barro and Gordon (1983).

More recently some authors-Swank (1994), Pearce and Sobue (1997), Schellekens (2002), and others-have argued that Brainard uncertainty ${ }^{2}$ is welfare-enhancing since policy caution is shown to reduce the inflation bias, providing an altogether different rationale for the benefits of caution: it is seen as a partial substitute for conservatism.

Schellekens (2002) pushes the debate further by arguing that, in the presence of Brainard uncertainty, a more careful specification of central bank preferences allows a distinction between two notions of conservatism termed 'stability-conservatism' and 'target-conservatism'. Whereas delegation of monetary policy to a Rogoff-style weight-conservative central banker partially eliminates the inflation bias at the expense of less output stabilization (a third-best outcome), stabilityconservatism, and target-conservatism can, in principle, eliminate the entire inflation bias without compromising the stabilization of output (a second-best outcome).

However, this recent strand of research on caution and conservatism suffers from a serious flaw: the output distortion responsible for the inflation bias is exogenous and constant. ${ }^{3}$ In a largely unrelated branch of the literature numerous authors challenge the conventional wisdom that monetary institutions only affect inflation and not the real economy. ${ }^{4}$ They show that when the labour market is populated by non-atomistic wage setters with market power, institutional characteristics do affect real variables.

This paper marries these two strands of the literature, allowing for a joint analysis of caution and central bank conservatism while endogenizing the output distortion that generates the time inconsistency problem. Two key results emerge: (i) for plausible parameter values, higher Brainard uncertainty raises the real wage premium and unemployment as well as the variances of inflation and unemployment, negating the anti-inflationary benefits of policy caution; and (ii) Schellekens's refined notions of conservatism are not able to generate the second-best outcome when there are non-atomistic wage setters.

\footnotetext{
${ }^{2}$ The terms Brainard uncertainty, parameter uncertainty and multiplicative uncertainty are used interchangeably.

${ }^{3}$ A notable exception is Lawler (2002) whose main result is a special case of Proposition 1, as discussed below.

${ }^{4}$ These include, but are not limited to, Akhand (1992), Cubitt (1992), Skott (1997), Guzzo and Velasco (1999), Cukierman and Lippi (1999, 2005), Soskice and Iversen (2000), Lawler (2000, 2002), and Lippi (2003).
} 


\section{Model setup}

\subsection{The labour market}

The following is an adaptation of Cukierman and Lippi (1999) (henceforth CL) who model an economy consisting of $n$ identical independent inflation-averse monopolistic unions, each facing the loss function

$$
\Omega_{j}=E\left\{-2 w_{j}+\chi u_{j}^{2}+\xi \pi^{2}\right\} ; \quad \chi>0 ; \quad \xi \geqslant 0
$$

where $w_{j}$ is the (logarithm of the) real wage received by union $j$ 's members, $u_{j}$ is the unemployment rate among members of union $j, \pi=p-p_{-1}$ is the rate of inflation where $p$ is the log of the price level, and $E$ is the standard expectations operator conditional on previous period's information. ${ }^{5}$ Labour is imperfectly substitutable across unions and supplied inelastically. All workers are unionized and evenly distributed across the $n$ unions. The demand for labour facing union $j$ is given by

$$
L_{j}^{d}=\left[\frac{\alpha}{n}\left(d-w_{j}\right)-\gamma\left(w_{j}-\bar{w}\right)\right] L ; \quad \alpha>0 ; \quad d>1 / \alpha
$$

where $L$ denotes total labour supply in the economy and $\bar{w}=\sum_{j=1}^{n} w_{j} / n$ is the economy-wide mean of $w_{j}$ across all unions. Such a labour demand function can be derived from a firm's standard profit maximization problem where the production function is characterized by constant or decreasing returns to scale. ${ }^{6}$

As in CL, aggregate demand for labor is

$$
L^{d}=\sum_{j=1}^{n} L_{j}^{d}=\alpha(d-\bar{w}) L .
$$

The economy-wide unemployment rate, absent any shocks, is defined as

$$
\widehat{u} \equiv \frac{L-L^{d}}{L}=\alpha\left(\bar{W}-\pi-p_{-1}-w^{c}\right)
$$

where $\bar{W}$ is the economy-wide mean of the (log) nominal wage and $w^{c} \equiv d-1 / \alpha$ is the (theoretical) market clearing wage that would generate an unemployment rate of zero. Actual unemployment is given by

$$
u=\widehat{u}+\varepsilon=\alpha\left(\bar{W}-\pi-p_{-1}-w^{c}\right)+\varepsilon
$$

where $\varepsilon$ is a macro-shock with mean zero and variance $\sigma_{\varepsilon}^{2}$ which the central bank observes but unions do not.

\footnotetext{
${ }^{5}$ For thorough discussions of trade union preferences see Oswald (1982), Layard et al. (1991), Cukierman and Lippi (1999), and Cahuc and Zylberberg (2004). For a justification of including the inflation term in the unions' loss function see Skott (1997).

${ }^{6}$ This derivation is not particularly illuminating. Interested readers are referred to Guzzo and Velasco (1999) and Coricelli et al. (2006) for examples of the derivation of similar labour demand schedules.
} 


\subsection{The central bank}

The modelling of the central bank's problem closely follows Swank (1994) and Schellekens (2002). The central bank minimizes the following generalized quadratic loss function:

$$
L=\mu_{1}\{E[\pi]\}^{2}+\theta_{1} \operatorname{Var}[\pi]+\mu_{2}\{E[u]\}^{2}+\theta_{2} \operatorname{Var}[u] .
$$

This loss function separates the costs of expected and unexpected deviations of inflation and output. In particular, the parameters $\mu_{1}$ and $\mu_{2}$ measure the intensity of the policy maker's aversion to systematically missing the inflation and output targets, respectively. The parameters $\theta_{1}$ and $\theta_{2}$ respectively gauge the policy maker's relative preference for nominal and real stability. The standard quadratic loss function is a special case with $\mu_{1}=\theta_{1}=\eta$, some non-negative number, and $\mu_{2}=\theta_{2}=1$. The benefit of using the generalized quadratic loss function will become apparent later.

Inflation is given by

$$
\pi=s i
$$

where $s$, the control error, is an iid random variable with positive support, a mean of unity, and finite variance $\sigma_{s}^{2}$, and $i$ represents the deviation of the central bank's monetary policy instrument from its neutral level, viz. that level which, absent any shocks, keeps the inflation rate constant at zero. Note that while only the central bank observes $\varepsilon$, neither it nor the unions observe $s$.

The quadratic nature of the loss function implies that the central bank's optimal policy rule will take the linear form

$$
i=\Lambda_{1}+\Lambda_{2} \varepsilon,
$$

where $\Lambda_{1}$ and $\Lambda_{2}$, yet to be determined, represent the central bank's control variables.

\section{Equilibrium}

In this game unions play Nash with each other but collectively act as Stackelberg leader vis-à-vis the central bank. Hence, the timing of events is as follows: First, unions set nominal wages. Second, the supply shock is realized, after which the central bank chooses monetary policy. Finally, the control error is realized and the unions and the central bank receive their respective payoffs.

\subsection{Solution to central bank's problem}

The model is solved by backward induction. The central bank's problem is to minimize (6) subject to (5), taking $\bar{W}$ as given. The optimal solution for $\Lambda_{1}$ is given by

$$
\Lambda_{1}=\frac{\mu_{2} \alpha^{2}}{\mu_{1}+\mu_{2} \alpha^{2}+\sigma_{s}^{2}\left(\theta_{1}+\theta_{2} \alpha^{2}\right)}(\bar{\phi}+E[\pi]),
$$


which makes use of the relationship $\bar{W}=\bar{w}+E[p]$ and of the definition of the real wage premium, $\bar{\phi} \equiv \bar{w}-w^{c}$. Noting that $E[\pi]=E[s i]=\Lambda_{1}$, eliminating $E[\pi]$ from (9) gives $\Lambda_{1}$ in equilibrium: ${ }^{7}$

$$
\Lambda_{1}=\frac{\mu_{2} \alpha^{2}}{\mu_{1}+\sigma_{s}^{2}\left(\theta_{1}+\theta_{2} \alpha^{2}\right)} \bar{\phi} .
$$

Setting $\mu_{1}=\theta_{1}=\eta, \mu_{2}=\theta_{2}=1$, and $\sigma_{s}^{2}=0$ replicates the result in CL.

The solution for $\Lambda_{2}$ is

$$
\Lambda_{2}=\frac{\alpha \theta_{2}}{\left(1+\sigma_{s}^{2}\right)\left(\theta_{1}+\theta_{2} \alpha^{2}\right)}
$$

\subsection{Solution to unions' problem}

Unions solve a standard optimization problem, setting nominal wages based on expectations of future inflation. Hence, union $j$ minimizes (1) subject to the central bank's reaction function (9) and its labour demand function (2) while taking other unions' wages as given. Making use of the definition of union j's unemployment rate, given by

$$
u_{j} \equiv \frac{L_{j}-L_{j}^{d}}{L_{j}}=\alpha\left(W_{j}-\pi-p_{-1}-w^{c}\right)+\gamma n\left(W_{j}-\bar{W}\right),
$$

the solution to union $j$ 's problem, after imposing symmetry and rearranging, yields the following expression for the economy-wide real wage premium: ${ }^{8}$

$$
\bar{\phi}=\frac{\mu_{2} \alpha^{2}(n-1)+\lambda n}{\chi \alpha^{2}\left(\mu_{2} \alpha^{2}(n-1)+\lambda n\right)+\chi \alpha \gamma n(n-1)\left(\lambda+\mu_{2} \alpha^{2}\right)+\Phi \frac{\mu_{2}^{2} \alpha^{4}}{\lambda}}
$$

with $\lambda \equiv \mu_{1}+\sigma_{s}^{2}\left(\theta_{1}+\theta_{2} \alpha^{2}\right)$ and $\Phi \equiv \xi+\sigma_{s}^{2}\left(\xi+\chi \alpha^{2}\right)$.

The expected equilibrium unemployment rate in the economy is given by

$$
E[u]=\alpha \bar{\phi},
$$

while average inflation is given by (10). Quick inspection of (13), (14), and (10) reveals that the real wage premium, the unemployment rate, and inflation are strictly positive.

\footnotetext{
${ }^{7} \mathrm{~A}$ more detailed derivation of the central bank's reaction function is offered in Appendix 1.

${ }^{8} \mathrm{~A}$ detailed derivation of eq. (13) is provided in Appendix 2.
} 


\section{Discussion}

\subsection{Caution}

How are the real wage premium and average unemployment affected by the presence of Brainard uncertainty? The following proposition gives an answer:

\section{Proposition 1}

(i) If $\quad \mu_{1}\left(\xi+\chi \alpha^{2}\right)>\xi\left(\theta_{1}+\theta_{2}\right)+\left(\theta_{1}+\theta_{2}\right) \mu_{1}\left(\chi \gamma n+(n-1)+\mu_{2} \alpha \xi n\right) /\left(\mu_{2} \alpha^{2}\right.$ $\left.(n-1)+\mu_{1} n\right) \mu_{2} \alpha$, an increase in Brainard uncertainty (a higher $\sigma_{s}^{2}$ ) lowers the equilibrium real wage premium and average unemployment at low $\sigma_{s}^{2}$ and raises the equilibrium real wage premium and average unemployment at high $\sigma_{s}^{2}$.

(ii) If $\mu_{1}\left(\xi+\chi \alpha^{2}\right)<\xi\left(\theta_{1}+\theta_{2}\right)+\left(\theta_{1}+\theta_{2}\right) \mu_{1}\left(\chi \gamma n(n-1)+\mu_{2} \alpha \xi n\right) /\left(\mu_{2} \alpha^{2}(n-1)+\right.$ $\left.\mu_{1} n\right) \mu_{2} \alpha$, an increase in Brainard uncertainty (a higher $\sigma_{s}^{2}$ ) monotonically raises the equilibrium real wage premium and average unemployment.

(iii) For sufficiently large $n$ an increase in Brainard uncertainty (a higher $\sigma_{s}^{2}$ ) monotonically raises the equilibrium real wage premium and average unemployment.

(iv) As $\sigma_{s}^{2} \rightarrow \infty$, the real wage premium $\bar{\phi} \rightarrow\left(\chi \alpha^{2}+\chi \alpha \gamma n(n-1)\right)^{-1}$.

\section{Proof See Appendix 3.}

With Brainard uncertainty unions face a trade-off between their real wage objective and unemployment stability. They are able to reduce the variance of inflation and unemployment at the expense of lower wages (which also implies lower mean inflation).

Thus case (i) highlights that there are two effects at work-the 'mean effect' and the 'variance effect'. On the one hand, unions are aware that increased uncertainty makes the central bank more cautious, leading to a reduction in mean inflation. Given that they have some monopoly power and they are Stackelberg leaders in the policy game, they want to exploit the central bank's restraint by increasing nominal wages. In other words, unions know that the central bank will inflate away less of any nominal wage increase because it fears the implications of strong activist policy.

On the other hand, greater uncertainty, for low values of $\sigma_{s}^{2}$ and any given value of $\bar{\phi}$, increases the variance of inflation and unemployment, forcing unions to show wage restraint. In case (i) the variance effect dominates the mean effect for small $\sigma_{s}^{2}$ and vice versa for large $\sigma_{s}^{2}$. In case (ii), the mean effect always dominates the variance effect.

Ceteris paribus, case (ii) obtains when unions care sufficiently about inflation relative to real wages and unemployment (high $\xi$ ) and when the central bank cares little about mean inflation relative to all its other objectives (low $\mu_{1}$ and 
high $\mu_{2}, \theta_{1}, \theta_{2}$ ). In this case the mean effect is so strong that it always dominates the variance effect.

Furthermore, the mean effect globally dominates the variance effect when the number of unions $n$ is large. An increase in the number of unions has two opposite effects: each union's market power falls (since the elasticity of labour demand facing each union rises), putting downward pressure on real wages-the 'competition effect'-but each union internalizes less the extent to which its own actions affect aggregate prices, putting upward pressure on real wages- the 'strategic effect.' above, increasing the range of parameter values for which case (ii) applies. As the number of unions goes to infinity, each union's monopoly power vanishes and the real wage premium disappears. Unsurprisingly, the wage premium is then independent of $\sigma_{s}^{2}$ and of unions' and central bank's preference parameters.

Proposition 1 generalizes the results contained in Lawler (2002). His model assumes a single monopoly union whose preferences do not include inflation. Setting $n=1, \xi=0, \chi=1, \mu_{1}=\theta_{1}=\eta$, and $\mu_{2}=\theta_{2}=1$ eq. (13) becomes

$$
\bar{\phi}=\frac{\left[\eta+\sigma_{s}^{2}\left(\eta+\alpha^{2}\right)\right]^{2}}{\alpha^{2}\left(\left[\eta+\sigma_{s}^{2}\left(\eta+\alpha^{2}\right)\right]^{2}+\sigma_{s}^{2} \alpha^{4}\right)},
$$

which is analogous to Lawler's expression. With these parameter values the real wage premium is indeed U-shaped, attaining a minimum at $\sigma_{s}^{2}=\eta /\left(\alpha^{2}+\eta\right)$. Lawler concludes that this is a general result since he assumes that, 'the key results derived below extend to a multi-union setting providing each union is sufficiently large to have some impact on macroeconomic outcomes' (Lawler, 2002, p.37). Proposition 1 shows that Lawler's conclusion is rather more specific.

The above point is not merely a theoretical curiosity. There is good reason to believe that case (ii) is actually the empirically dominant one. For reasonable parameter values it only takes a small number of unions to generate case (ii). For example, when $\alpha=0.7, \gamma=1, \mu_{1}=\theta_{1}=2, \mu_{2}=\theta_{2}=1, \xi=0.4$ and there is only one all-encompassing monopoly union, the real wage premium is monotonically increasing in $\sigma_{s}^{2}$, viz. case (ii) obtains. One may well argue that $\xi=0.4$ is too high. Lowering it to 0.1 breaks the monotonicity of $\bar{\phi}$ (viz. generates case (i)) but as soon as there are two or more unions, the monotonicity is restored. Since most economies are populated by several unions, it is very difficult to generate case (i) unless one assumes extreme values for some of the parameters.

Several authors have argued that multiplicative uncertainty in the policy transmission enhances welfare because it makes the policymaker more cautious

\footnotetext{
${ }^{9}$ The joint presence of these two effects gives rise to the well documented hump-shaped relation between the degree of centralization of wage bargaining and real wages. See Calmfors and Driffill (1988), Calmfors (1993), and Cukierman and Lippi (1999). This is confirmed by differentiating eq. (13) with respect to $n$.
} 
and thereby reduces the inflation bias. ${ }^{10}$ Proposition 1 shows that, for reasonable parameter values, an increase in multiplicative uncertainty increases the wage premium, leading to a bigger unemployment distortion. This, in turn, raises the incentive for the central bank to engineer an inflation surprise. The reason why Proposition 1 departs from previous authors' conclusions is that their analysis is based on an invariant output distortion. With monopolistic unions the output (or unemployment) distortion is endogenous and thus becomes a function of the economic environment and therefore also of the degree of uncertainty. ${ }^{11}$

If the central bank loss function (6) is taken to be the appropriate social welfare function, then an increase in Brainard uncertainty has an ambiguous effect on welfare. Caution resulting from Brainard uncertainty is not, in general, welfare enhancing. ${ }^{12}$

Proposition 1 is also very different from the argument advanced by Sørensen (1991), Grüner (2002), and Grüner et al. (2005). In their models uncertainty about central bank preferences unambiguously reduces wages, average inflation, and unemployment. The central bank does not face parameter uncertainty and therefore sets optimal policy in a standard certainty-equivalent framework. Once the central bank reaction function is obtained, the associated elasticities are assumed to be random. This is supposed to capture the notion that unions are unsure about the central bank's preferences. Here, on the other hand, the central bank's control of the monetary policy process is imprecise which tempers the response to wage claims and thus leads to higher wages and lower unemployment.

\subsection{Conservatism}

The following result summarizes the comparative statics with regard to the central bank's preference parameters:

Proposition 2 An increase in $\mu_{1}, \theta_{1}$, and $\theta_{2}$ and a decrease in $\mu_{2}$ all raise the equilibrium real wage premium and average unemployment. These effects become negligible as $n \rightarrow \infty$.

\footnotetext{
${ }^{10}$ For example, Swank (1994, p.30) argues that, 'in a stochastic framework, it appears that an increase in multiplicative uncertainty about policy effects on money growth reduces the policymaker's incentive to create inflation surprises and increases welfare'. See also Pearce and Sobue (1997) and Schellekens (2002) for a similar sentiment.

${ }^{11}$ Output and unemployment, as modelled here, are inversely related. It is easy to rephrase the present model in terms of output instead of unemployment.

${ }^{12}$ Letterie (1997) argues that when the policymaker has private information about an economic shock, higher uncertainty is costly. Thus, the benefits of Brainard uncertainty (smaller inflation bias) may be outweighed by its costs (less stabilization).
} 
Proof Differentiating (13) with respect to $\mu_{1}, \theta_{1}, \theta_{2}$, and $\mu_{2}$ gives

$$
\frac{\partial \bar{\phi}}{\partial \mu_{1}}=Y>0, \quad \frac{\partial \bar{\phi}}{\partial \theta_{1}}=\sigma_{s}^{2} Y>0, \quad \frac{\partial \bar{\phi}}{\partial \theta_{2}}=\sigma_{s}^{2} \alpha^{2} Y>0,
$$

where

$$
Y \equiv \frac{\chi \mu_{2} \alpha \gamma n(n-1)+\Phi \frac{\mu_{2}^{2} \alpha^{2}}{\lambda}\left(2 n+\frac{\mu_{2} \alpha^{2}(n-1)}{\lambda}\right)}{\left[\chi \alpha\left(\mu_{2} \alpha^{2}(n-1)+\lambda n\right)+\chi \gamma n(n-1)\left(\lambda+\mu_{2} \alpha^{2}\right)+\Phi \frac{\mu_{2}^{2} \alpha^{3}}{\lambda}\right]^{2}}>0
$$

and

$$
\frac{\partial \bar{\phi}}{\partial \mu_{2}}=-\frac{\chi \alpha \gamma n(n-1) \lambda+\Phi \frac{\mu_{2} \alpha^{2}}{\lambda}\left(\mu_{2} \alpha^{2}(n-1)+2 \lambda n\right)}{\left[\chi \alpha\left(\mu_{2} \alpha^{2}(n-1)+\lambda n\right)+\chi \gamma n(n-1)\left(\lambda+\mu_{2} \alpha^{2}\right)+\Phi \frac{\mu_{2}^{2} \alpha^{3}}{\lambda}\right]^{2}}<0 .
$$

The intuition for this result is as follows. When $\mu_{1}$ is high, the monetary authority places a lot of emphasis on hitting the inflation target. She will therefore be loath to inflate away the high wages set by the unions. The latter take advantage of this, and the real wage premium will be relatively high. Conversely, when $\mu_{2}$ is high, the monetary authority has a strong preference for low average unemployment. It is willing to accept higher inflation in order to erode the excessive nominal wages set by the unions. The real wage premium will be relatively small, as unions know that high wages are inflated away.

The effects of $\theta_{1}$ and $\theta_{2}$ on $\bar{\phi}$ are similarly intuitive. When $\theta_{1}\left(\theta_{2}\right)$ increases, the policymaker places more emphasis on avoiding unforecastable deviations in inflation (unemployment) from target. A higher $\sigma_{s}^{2}$ implies that monetary policy itself injects more uncertainty into the system. Thus, fearing the uncertain consequences of its own actions, the monetary authority becomes reluctant to generate high inflation in response to high nominal wages. As a result, the real wage premium will be relatively large.

When $\sigma_{s}^{2}=0$, the real wage premium no longer depends on $\theta_{1}$ and $\theta_{2}$. Likewise, when $n \rightarrow \infty$, unions neglect the effect of their own actions on inflation so that the wage premium becomes independent of the policymaker's preferences. ${ }^{13}$

Proposition 2 is important when assessing the validity of the existing literature on caution and conservatism in monetary policy as conventional results are

\footnotetext{
${ }^{13}$ The benefit of using the generalized loss function for the government should be clear by now. The conventional loss function, such as the one used by CL, imposes $\mu_{1}=\theta_{1}=\eta$ and $\mu_{2}=\theta_{2}=1$ and obscures the opposing effects of $\mu_{2}$ and $\theta_{2}$ on the real wage premium.
} 
overturned when the output/unemployment distortion is endogenized. For example, Schellekens argues that,

... delegation to a conservative central banker does not entail suboptimal output stabilization if conservatism is not arbitrarily restricted to the notion of weightconservatism. Alternative forms of conservatism, such as stability-conservatism and target-conservatism, reduce the inflationary bias without distorting the stabilization of output. [...] Any delegation scheme which improves or removes the credibility problem of monetary policy reduces at the same time the variability of output (and of inflation), if the transmission of monetary policy is subject to multiplicative uncertainty.

The overall theoretical implication is thus, surprisingly, that delegation based on optimal notions of conservatism should not only lead to lower inflation but also to less variable output. (Schellekens, 2002, p.173) ${ }^{14}$

The generalization of this statement is inappropriate for two reasons. First, Schellekens claims that delegation of monetary policy to a central banker with an exclusive concern for stability leads to the second-best outcome. This is modelled by assuming that the central bank has preference parameters $\theta_{1}^{*}$ and $\theta_{2}^{*}$ which are greater than society's $\theta_{1}$ and $\theta_{2}$ by a factor $\kappa^{*}$, e.g. $\theta_{1}^{*}=\kappa^{*} \theta_{1}$ and $\theta_{2}^{*}=\kappa^{*} \theta_{2}$ with $\kappa^{*}>1$. He shows that a higher $\kappa^{*}$ unambiguously reduces the inflation bias while leaving the stabilization component $\left(\Lambda_{2}\right)$ unchanged. Hence, choosing $\kappa^{*} \rightarrow \infty$ eliminates the inflation bias without distorting the stabilization component. However, this result crucially hinges on a constant output distortion. In the model presented here, delegating monetary policy to a central banker with $\kappa^{*}$ implies that average unemployment becomes

$$
E[u]=\alpha \bar{\phi}=\frac{\mu_{2} \alpha^{2}(n-1)+\lambda^{*} n}{\chi \alpha\left(\mu_{2} \alpha^{2}(n-1)+\lambda^{*} n\right)+\chi \gamma n(n-1)\left(\lambda^{*}+\mu_{2} \alpha^{2}\right)+\Phi \frac{\mu_{2}^{2} \alpha^{3}}{\lambda^{*}}}
$$

with $\lambda^{*} \equiv \mu_{1}+\kappa^{*} \sigma_{s}^{2}\left(\theta_{1}+\theta_{2} \alpha^{2}\right)$. It is straightforward to show that $\partial E[u] / \partial \kappa^{*}>0$. Thus, delegating policy to a stability-conservative central banker may well reduce or eliminate the inflation bias but at the cost of higher average unemployment. ${ }^{15}$

Second, contrary to Schellekens' claim, stability- and targetconservatism may adversely affect output/unemployment variability when the

\footnotetext{
${ }^{14}$ Weight-conservatism refers to the relative preference for inflation versus output stabilization. This is the traditional definition of conservatism, as in Rogoff (1985). Schellekens (2002) introduces the notions of stability-conservatism and target-conservatism based on the generalized quadratic objective function. Stability-conservatism is defined as a relative preference for nominal and real stability and corresponds to a higher $\theta_{1}$ and $\theta_{2}$. A central banker is said to be target-conservative if, relative to the government, she places more emphasis on hitting the inflation target than the output target. This corresponds to a relatively high $\mu_{1}$ and a relatively low $\mu_{2}$.

${ }^{15}$ Schellekens (2002) shows that target conservatism, defined as $\mu_{1}^{*} / \mu_{2}^{*}>\mu_{1} / \mu_{2}$, is also welfare improving and in the limit $\left(\mu_{2}^{*} \rightarrow 0\right)$ delivers the second-best outcome. However, Proposition 2 applies here as well: a lower $\mu_{2}$ raises the real wage premium and thus raises the incentive to inflate, preventing the attainment of the second-best outcome.
} 
output/unemployment distortion is endogenous. Consider the variance of unemployment, which, in equilibrium, is given by

$$
\begin{aligned}
\operatorname{Var}[u]= & \left(\frac{\mu_{2} \alpha^{3} \bar{\phi}}{\lambda}\right)^{2} \sigma_{s}^{2} \\
& +\left(1+\left(1+\sigma_{s}^{2}\right)\left(\frac{\theta_{2} \alpha^{2}}{\left(1+\sigma_{s}^{2}\right)\left(\theta_{1}+\theta_{2} \alpha^{2}\right)}\right)^{2}-\frac{2 \theta_{2} \alpha^{2}}{\left(1+\sigma_{s}^{2}\right)\left(\theta_{1}+\theta_{2} \alpha^{2}\right)}\right) \sigma_{\varepsilon}^{2} .
\end{aligned}
$$

More inflation stability-conservatism (a higher $\theta_{1}$ ) increases the real wage premium, thereby increasing the unemployment distortion (Proposition 2). Equation (16) indicates that this leads to higher unemployment variability in equilibrium since it is easily shown that $d \operatorname{Var}[u] / d \theta_{1}=(\partial \operatorname{Var}[u] / \partial \bar{\phi})\left(\partial \bar{\phi} / \partial \theta_{1}\right)+$ $\partial \operatorname{Var}[u] / \partial \theta_{1}>0$.

Proposition 2 shows that even refined notions of conservatism are not able to deliver a second-best outcome: with non-atomistic wage setters both optimal target conservatism and optimal stability conservatism increase the real wage premium while possibly also increasing the variance of unemployment.

\section{Conclusion}

Recent research suggested that society benefits from delegating monetary policy to a cautious and conservative central banker. In particular, caution was seen as a partial substitute for conservatism, helping to reduce the costly inflation bias, while an optimal notion of conservatism was shown to deliver a secondbest outcome, entirely eliminating the inflation bias without compromising the stabilization of output.

This paper highlighted the severe limitations of this research, arguing that the strong welfare implications of this literature are predicated on an invariant output distortion. It was shown that when wages are set by monopolistic unions, an increase in multiplicative uncertainty-for reasonable parameter values-increases the real wage premium, leading to a bigger unemployment distortion, which in turn raises the incentive for the central bank to engineer an inflation surprise. This suggests that policy caution resulting from Brainard uncertainty does not increase welfare. Furthermore, since central bank preferences also affect real variables, delegating policy to an independent central banker with an optimal degree of conservatism cannot, in general, deliver a second-best outcome.

The present model is limited by its static nature. A dynamic analysis would be able to account for the possibility that optimality of monetary policy under multiplicative uncertainty calls for greater aggressiveness and therefore a worsening of the inflation bias. ${ }^{16}$ Furthermore, a richer environment, such as a New Keynesian

${ }^{16}$ See, for example, Craine (1979) and Söderström (2002). 
model with nominal wage rigidities, would yield further insights and set the stage for a thorough empirical analysis. These challenges are left for the future.

The results in this paper provide a powerful reminder that one should refrain from hastily drawing policy conclusions when the model does not adequately specify the private sector. Policy makers should heed Skott's general concerns about this literature:

... It seems unfortunate that Barro-Gordon type models have been so influential.

The conclusions from these models are sensitive to the precise specification and...the basic Barro-Gordon set-up even supports policies that are the exact opposite of those normally asociated with this literature. (Skott, 1997, p.620)

\section{Acknowledgements}

Much of this paper was written while visiting the Humboldt University Berlin in 2006. I am grateful to Robin Cubitt, Alex Heath, Timothy Kam, Ben Martin, Warwick McKibbin, Philip Schellekens, Geoff Shuetrim, and three anonymous referees for helpful comments. All remaining errors are my own.

\section{Funding}

Deutsche Forschungsgemeinschaft (SFB 649 'Economic Risk').

\section{References}

Akhand, H.A. (1992) Policy credibility and inflation as a wage-setting game, Canadian Journal of Economics, 25, 407-19.

Barro, R.J. and Gordon, D.B. (1983) A positive theory of monetary policy in a natural rate model, Journal of Political Economy, 91, 589-610.

Blinder, A.S. (1998) Central Banking in Theory and Practice, MIT Press, Cambridge, MA.

Brainard, W.C. (1967) Uncertainty and the effectiveness of policy, American Economic Review, 57, 411-25.

Cahuc, P. and Zylberberg, A. (2004) Labor Economics, MIT Press, Cambridge, MA.

Calmfors, L. (1993) Centralisation of wage bargaining and macroeconomic performance-a survey, OECD Economic Studies No. 21, 161-91.

Calmfors, L. and Driffill, J. (1988) Bargaining structure, corporatism, and macroeconomic performance, Economic Policy, 6, 14-61.

Coricelli, F., Cukierman, A., and Dalmazzo, A. (2006) Monetary institutions, monopolistic competition, unionised labor markets and economic performance, Scandinavian Journal of Economics, 108, 39-63.

Craine, R. (1979) Optimal montary policy with uncertainty, Journal of Economic Dynamics and Control, 1, 59-83.

Cubitt, R.P. (1992) Monetary policy games and private sector precommitment, Oxford Economic Papers, 44, 513-30. 
Cukierman, A. and Lippi, F. (1999) Central bank independence, centralisation of wage bargaining, inflation and unemployment, European Economic Review, 43, 1395-434.

Grüner, H.P. (2002) How much should central banks talk? A new argument, Economics Letters, 77, 196-98.

Grüner, H.P., Hayo, B., and Hefeker, C. (2005) Unions, wage setting and monetary policy uncertainty, Working Paper No. 490, June, European Central Bank, Frankfurt.

Guzzo, V. and Velasco, A. (1999) The case for a populist central banker, European Economic Review, 43, 1317-44.

Kydland, F.E. and Prescott, E.C. (1977) Rules rather than discretion: the inconsistency of optimal plans, Journal of Political Economy, 85, 473-92.

Lawler, P. (2000) Centralised wage setting, inflation contracts, and the optimal choice of central banker, Economic Journal, 110, 559-75.

Lawler, P. (2002) Monetary uncertainty, strategic wage setting and equilibrium employment, Economics Letters, 77, 35-40.

Layard, R., Nickell, S., and Jackman, R. (1991) Unemployment-Macroeconomic Performance and the Labour Market, Oxford University Press, Oxford.

Letterie, W. (1997) Better monetary control may decrease the distortion of stabilisation policy: a comment, Scandinavian Journal of Economics, 99, 463-70.

Lippi, F. (2003) Strategic monetary policy with non-atomistic wage setters, Review of Economic Studies, 70, 601-12.

Oswald, A.J. (1982) The microeconomic theory of trade unions, Economic Journal, 92, 576-95.

Pearce, D. and Sobue, M. (1997) Uncertainty and the inflation bias of monetary policy, Economics Letters, 57, 203-7.

Rogoff, K. (1985) The optimal degree of commitment to an intermediate target, Quarterly Journal of Economics, 100, 1169-90.

Schellekens, P. (2002) Caution and conservatism in the making of monetary policy, Journal of Money, Credit, and Banking, 34, 160-77.

Skott, P. (1997) Stagflationary consequences of prudent monetary policy in a unionized economy, Oxford Economic Papers, 49, 609-22.

Söderström, U. (2002) Monetary policy with uncertain parameters, Scandinavian Journal of Economics, 104, 125-45.

Sørensen, J.R. (1991) Political uncertainty and macroeconomic performance, Economics Letters, 37, 377-81.

Soskice, D. and Iversen, T. (2000) The non neutrality of monetary policy with large price or wage setters, Quarterly Journal of Economics, 115, 265-84.

Swank, O.H. (1994) Better monetary control may increase the inflationary bias of policy, Scandinavian Journal of Economics, 96, 125-31. 


\section{Appendix 1}

\section{Derivation of central bank's reaction function}

By substituting (5), (7), and (8) into (6), the central bank's objective function may be rewritten as

$$
\begin{aligned}
L= & \mu_{1} \Lambda_{1}^{2}+\theta_{1}\left(\Lambda_{1}^{2} \sigma_{s}^{2}+\Lambda_{2}^{2} \sigma_{\varepsilon}^{2}\left(1+\sigma_{s}^{2}\right)\right)+\mu_{2}\left(\alpha \bar{W}-\alpha \Lambda_{1}-\alpha p_{-1}-\alpha w^{c}\right)^{2} \\
& +\theta_{2}\left(\Lambda_{1}^{2} \alpha^{2} \sigma_{s}^{2}+\Lambda_{2}^{2} \alpha^{2} \sigma_{\varepsilon}^{2}\left(1+\sigma_{s}^{2}\right)+\sigma_{\varepsilon}^{2}-2 \alpha \Lambda_{2} \sigma_{\varepsilon}^{2}\right)
\end{aligned}
$$

which makes use of the statistical relationship $\operatorname{Var}[s \varepsilon]=E[\varepsilon] \operatorname{Var}[s]+E[s] \operatorname{Var}[\varepsilon]+$ $\operatorname{Var}[\varepsilon] \operatorname{Var}[s]$ (since $\varepsilon$ and $s$ are independent).

Differentiating the central bank's objective function with respect to $\Lambda_{1}$ and setting equal to zero gives the central bank's first-order condition,

$$
\mu_{1} \Lambda_{1}+\theta_{1} \Lambda_{1} \sigma_{s}^{2}+\theta_{2} \Lambda_{1} \alpha^{2} \sigma_{s}^{2}=\mu_{2} \alpha\left(\alpha \bar{W}-\alpha \Lambda_{1}-\alpha p_{-1}-\alpha w^{c}\right)
$$

which, by making use of the relationships $\bar{W}=\bar{w}+E[p], \bar{\phi} \equiv \bar{w}-w^{c}$, and $E[\pi]=\Lambda_{1}$, may be solved for $\Lambda_{1}$, eq. (10) in the text.

\section{Appendix 2}

\section{Derivation of real wage premium}

Substituting (12) into (1), the union's minimization problem becomes

$$
\min _{W_{j}} E\left\{-2\left(W_{j}-\pi-p_{-1}\right)+\chi\left[\alpha\left(W_{j}-\pi-p_{-1}-w^{c}\right)+\gamma n\left(W_{j}-\bar{W}\right)\right]^{2}+\xi \pi^{2}\right\},
$$

which makes use of $w_{j}=W_{j}-E[p]=W_{j}-E[p]+p_{-1}-p_{-1}=W_{j}-E[\pi]-p_{-1}$.

Replacing $\pi$ with (7) and (8), differentiating the above expression with respect to $W_{j}$, and setting equal to zero gives the union's first-order condition:

$$
E\left\{1-s \frac{\partial \Lambda_{1}}{\partial W_{j}}\right\}=E\left\{\chi u_{j}\left(\alpha-\alpha s \frac{\partial E[\pi]}{\partial W_{j}}+\gamma(n-1)\right)\right\}+E\left\{\xi \pi s \frac{\partial \Lambda_{1}}{\partial W_{j}}\right\}
$$

Making use of the following relationship, $\phi_{j}=w_{j}-w^{c}=W_{j}-E[p]-w^{c}=$ $W_{j}-E[\pi]-p_{-1}-w^{c}$ and defining $Z \equiv 1-\partial \Lambda_{1} / \partial W_{j}$, the above FOC can be simplified to

$$
\chi\left[\alpha \phi_{j}+\gamma n\left(\phi_{j}-\bar{\phi}\right)\right](\alpha Z+\gamma(n-1))+(1-Z) \Lambda_{1}\left(\xi+\sigma_{s}^{2}\left(\xi+\chi \alpha^{2}\right)\right)=Z .
$$

Imposing symmetry $\left(\phi_{j}=\phi_{i}=\bar{\phi}\right)$ gives

$$
\chi \alpha \bar{\phi}(\alpha Z+\gamma(n-1))+(1-Z) \Lambda_{1}\left(\xi+\sigma_{s}^{2}\left(\xi+\chi \alpha^{2}\right)\right)=Z
$$

which can be solved for $\bar{\phi}$ to give

$$
\bar{\phi}=\frac{Z-(1-Z) \Lambda_{1}\left(\xi+\sigma_{s}^{2}\left(\xi+\chi \alpha^{2}\right)\right)}{\chi \alpha(\alpha Z+\gamma(n-1))} .
$$


Substituting for $Z$ and $\Lambda_{1}$ and simplifying gives the equilibrium real wage premium, eq. (13) in the text.

\section{Appendix 3}

Proof of Proposition 1 (i) and (ii) Differentiating (13) with respect to $\sigma_{s}^{2}$ yields

$$
\begin{aligned}
\frac{\partial \bar{\phi}}{\partial \sigma_{s}^{2}}= & \frac{1}{D^{2}}\left\{\left(\theta_{1}+\theta_{2} \alpha^{2}\right) \mu_{2} \alpha^{3}[\chi \gamma n(n-1)\right. \\
& \left.+\left(\xi+\sigma_{s}^{2}\left(\xi+\chi \alpha^{2}\right)\right) \frac{\mu_{2} \alpha}{\lambda}\left(2 n+\frac{\mu_{2} \alpha^{2}(n-1)}{\lambda}\right)\right] \\
& \left.-\frac{\mu_{2}^{2} \alpha^{4}}{\lambda}\left(\xi+\chi \alpha^{2}\right)\left(\mu_{2} \alpha^{2}(n-1)+\lambda n\right)\right\}
\end{aligned}
$$

where $D$ is the denominator of (13).

As $D^{2}$ is always positive, the sign of the derivative is equal to the sign of the numerator. After multiplying $\partial \bar{\phi} / \partial \sigma_{s}^{2}$ by $\lambda^{2} / \lambda^{2}$, rewrite the numerator as

$$
\begin{aligned}
& \left(\theta_{1}+\theta_{2} \alpha^{2}\right) \mu_{2} \alpha^{3} \chi \gamma n(n-1) \lambda^{2}+\left(\theta_{1}+\theta_{2} \alpha^{2}\right) \mu_{2}^{2} \alpha^{4} \Phi\left(2 \lambda n+\mu_{2} \alpha^{2}(n-1)\right) \\
& \quad-\mu_{2}^{2} \alpha^{4} \lambda\left(\xi+\chi \alpha^{2}\right)\left(\mu_{2} \alpha^{2}(n-1)+\lambda n\right)
\end{aligned}
$$

The first two terms are positive, the third term negative for any $\sigma_{s}^{2}$. One can easily show that the second term grows at a faster rate than the third term. Differentiating these two terms with respect to $\sigma_{s}^{2}$ gives

$$
\begin{aligned}
& \left(\theta_{1}+\theta_{2} \alpha^{2}\right) \mu_{2}^{2} \alpha^{4}\left[\left(\xi+\chi \alpha^{2}\right)\left(2 \lambda n+\mu_{2} \alpha^{2}(n-1)\right)+\Phi 2 n\left(\theta_{1}+\theta_{2} \alpha^{2}\right)\right] \\
& \quad-\mu_{2}^{2} \alpha^{4}\left(\xi+\chi \alpha^{2}\right)\left[\left(\mu_{2} \alpha^{2}(n-1)+\lambda n\right)\left(\theta_{1}+\theta_{2} \alpha^{2}\right)+\lambda n\left(\theta_{1}+\theta_{2} \alpha^{2}\right)\right] \\
& \quad=\Phi 2 n\left(\theta_{1}+\theta_{2} \alpha^{2}\right)>0 .
\end{aligned}
$$

Thus, since the first term and the sum of the second and third terms are monotonically increasing in $\sigma_{s}^{2}$, the entire derivative $\left(\partial \bar{\phi} / \partial_{s}^{2}\right)$ becomes positive for sufficiently large $\sigma_{s}^{2}$.

Therefore, if $\partial \bar{\phi} / \partial \sigma_{s}^{2}>0$ at $\sigma_{s}^{2}=0$, then $\partial \bar{\phi} / \partial \sigma_{s}^{2}>0$ for all $\sigma_{s}^{2}$. If $\partial \bar{\phi} / \partial \sigma_{s}^{2}<0$ at $\sigma_{s}^{2}=0$, then $\bar{\phi}$ displays a U-shape, with a minimum at some finite $\sigma_{s}^{2}$.

At $\sigma_{s}^{2}=0$, (A1) becomes

$$
\begin{aligned}
& \left(\theta_{1}+\theta_{2} \alpha^{2}\right) \mu_{1}\left[\chi \gamma n(n-1) \mu_{1}+\mu_{2} \alpha \xi n\right] \\
& \quad+\left(\mu_{2} \alpha^{2}(n-1)+\mu_{1} n\right) \mu_{2} \alpha\left[\left(\theta_{1}+\theta_{2} \alpha^{2}\right) \xi-\mu_{1}\left(\xi+\chi \alpha^{2}\right)\right]
\end{aligned}
$$

This expression is negative when

$$
\mu_{1}\left(\xi+\chi \alpha^{2}\right)>\frac{\xi\left(\theta_{1}+\theta_{2}\right)+\left(\theta_{1}+\theta_{2}\right) \mu_{1}\left(\chi \gamma n(n-1)+\mu_{2} \alpha \xi n\right)}{\left(\mu_{2} \alpha^{2}(n-1)+\mu_{1} n\right) \mu_{2} \alpha} .
$$


From (14) it follows that the qualitative relation between expected unemployment and $\sigma_{s}^{2}\left(\partial E[u] / \partial \sigma_{s}^{2}\right)$ is the same as the qualitative relation between the real wage premium and $\sigma_{s}^{2}\left(\partial \bar{\phi} / \partial \sigma_{s}^{2}\right)$.

(iii) While (A2) may be negative it is always possible to find a sufficiently large $n$ to guarantee that (A2) is positive. To see this, differentiate (A2) with respect to $n$, which yields

$$
(2 n-1)\left(\theta_{1}+\theta_{2} \alpha^{2}\right) \chi \gamma \mu_{1}^{2}+\left(\mu_{2}^{2} \alpha^{3}+\mu_{1} \mu_{2} \alpha\right)\left[\left(\theta_{1}+\theta_{2} \alpha^{2}\right) \xi-\mu_{1}\left(\xi+\chi \alpha^{2}\right)\right] .
$$

This expression is monotonically increasing in $n$, implying that for some $n>n^{*}$ it becomes positive. Hence, for large enough $n$ the real wage premium $\bar{\phi}$ is monotonically increasing in $\sigma_{s}^{2}$.

(iv) Rewrite $\bar{\phi}$ as

$$
\bar{\phi}=\frac{\mu_{2} \alpha^{2}(n-1) \lambda+\lambda^{2} n}{\chi \alpha^{2}\left(\mu_{2} \alpha^{2}(n-1) \lambda+\lambda^{2} n\right)+\chi \alpha \gamma n(n-1)\left(\lambda^{2}+\mu_{2} \alpha^{2} \lambda\right)+\Phi \mu_{2}^{2} \alpha^{4}} \equiv \frac{p\left(\sigma_{s}^{2}\right)}{q\left(\sigma_{s}^{2}\right)} .
$$

Since $\lim _{\sigma_{s}^{2} \rightarrow \infty} p\left(\sigma_{s}^{2}\right)=\infty$ and $\lim _{\sigma_{s}^{2} \rightarrow \infty} q\left(\sigma_{s}^{2}\right)=\infty$, it is necessary to apply l'Hôpital's rule. Differentiating $p$ and $q$ with respect to $\sigma_{s}^{2}$ gives

$$
p^{\prime}\left(\sigma_{s}^{2}\right)=\mu_{2} \alpha^{2}(n-1)\left(\theta_{1}+\theta_{2} \alpha^{2}\right)+2\left(\mu_{1}+\sigma_{s}^{2}\left(\theta_{1}+\theta_{2} \alpha^{2}\right)\right)\left(\theta_{1}+\theta_{2} \alpha^{2}\right)
$$

and

$$
\begin{aligned}
q^{\prime}\left(\sigma_{s}^{2}\right)= & \chi \alpha^{2}\left[\mu_{2} \alpha^{2}(n-1)\left(\theta_{1}+\theta_{2} \alpha^{2}\right)+2\left(\mu_{1}+\sigma_{s}^{2}\left(\theta_{1}+\theta_{2} \alpha^{2}\right)\right)\left(\theta_{1}+\theta_{2} \alpha^{2}\right)\right] \\
& +\chi \alpha \gamma n(n-1)\left[2\left(\mu_{1}+\sigma_{s}^{2}\left(\theta_{1}+\theta_{2} \alpha^{2}\right)\right)\left(\theta_{1}+\theta_{2} \alpha^{2}\right)+\mu_{2} \alpha^{2}\left(\theta_{1}+\theta_{2} \alpha^{2}\right)\right] \\
& +\mu_{2}^{2} \alpha^{4}\left(\xi+\chi \alpha^{2}\right) .
\end{aligned}
$$

As $\lim _{\sigma_{s}^{2} \rightarrow \infty} p^{\prime}\left(\sigma_{s}^{2}\right)=\infty$ and $\lim _{\sigma_{s}^{2} \rightarrow \infty} q^{\prime}\left(\sigma_{s}^{2}\right)=\infty$, differentiate $p$ and $q$ again to obtain

$$
p^{\prime \prime}\left(\sigma_{s}^{2}\right)=2\left(\theta_{1}+\theta_{2} \alpha^{2}\right)^{2}
$$

and

$$
q^{\prime \prime}\left(\sigma_{s}^{2}\right)=2 \chi \alpha^{2}\left(\theta_{1}+\theta_{2} \alpha^{2}\right)^{2}+2 \chi \alpha \gamma n(n-1)\left(\theta_{1}+\theta_{2} \alpha^{2}\right)^{2} .
$$

Thus, according to l'Hôpital's rule,

$$
\lim _{\sigma_{s}^{2} \rightarrow \infty} \bar{\phi}=\lim _{\sigma_{s}^{2} \rightarrow \infty} \frac{p^{\prime \prime}\left(\sigma_{s}^{2}\right)}{q^{\prime \prime}\left(\sigma_{s}^{2}\right)}=\frac{1}{\chi \alpha^{2}+\chi \alpha \gamma n(n-1)} .
$$

